\title{
Bem-estar Subjetivo, Personalidade e Vivências Acadêmicas em Estudantes Universitários
}

\author{
Aline Bertuol Rosin \\ Universidade Federal do Rio Grande do Sul, Porto Alegre, Brasil \\ Cristian Zanon \\ Universidade São Francisco, Itatiba, Brasil \\ Marco Antônio Pereira Teixeira* \\ Universidade Federal do Rio Grande do Sul, Porto Alegre, Brasil
}

\begin{abstract}
RESUMO
O objetivo deste estudo foi investigar se vivências acadêmicas positivas estão associadas com o bemestar subjetivo (BES) de universitários, bem como as relações entre as vivências acadêmicas e a personalidade. Mais especificamente, procurou-se avaliar o quanto as variáveis de vivências acadêmicas predizem a variância do BES controlando o efeito dos fatores de personalidade. Participaram do estudo 227 universitários (59\% mulheres e 41\% homens). Os instrumentos utilizados foram um questionário sobre vivências acadêmicas, adjetivos marcadores reduzidos da personalidade, uma escala de satisfação de vida e uma escala de afetos positivos e negativos. Os resultados mostraram que a personalidade é o preditor mais saliente do bem-estar subjetivo. Porém, foram verificadas correlações significativas entre vivências acadêmicas e bem-estar subjetivo.
\end{abstract}

Palavras-chave: bem-estar subjetivo; vivências acadêmicas; personalidade; estudantes universitários.

\begin{abstract}
Subjective Well-being, Personality and Academic Experiences on University Students

The purpose of this study was to investigate possible associations between positive academic experiences and the subjective well-being of university students, as well as the relationships among academic experiences and personality factors. More specifically, it was assessed the contribution of academic experiences to the prediction of subjective well-being after controlling for the effects of personality. Participants were 227 college students (59\% women and $41 \%$ men). The instruments used were a questionnaire to evaluate academic experiences, reduced adjective markers of personality, the satisfaction with life scale, and the positive and negative affect scale. Results showed that personality is the best predictor of subjective well-being. However, significant correlations between academic experiences and subjective well-being were also observed.
\end{abstract}

Keywords: subjective well-being; academic experiences; personality; college students.

Geralmente a entrada na universidade significa a realização de um sonho que muitas vezes é compartilhado positiva e intensamente com as pessoas que fazem parte da vida do jovem recém-ingressado na vida acadêmica (Almeida \& Soares, 2004). Esses mesmos autores afirmam que os jovens, apesar de conseguirem ultrapassar várias barreiras, chegam à universidade com visões inocentes e irreais sobre o que realmente abarca a vida universitária, apresentando frequentemente durante o primeiro ano na vida acadêmica altos níveis de estresse e ansiedade que os deixam vulneráveis ao desajustamento e, em alguns casos, até a sintomas psicopatológicos (Lemos, 2010).

A transição para o ensino superior é indicativa de várias mudanças na vida do estudante, e seu impacto está relacionado às características pessoais e particulares de cada um, e também às exigências e apoios do contexto ao qual estão inseridos (Almeida, Soares \& Ferreira, 2002; Santos \& Almeida, 2001). Almeida e Soares (2004) apontam que a transição do ensino médio para o superior pode exigir muito dos jovens e confrontá-los com desafios novos e complexos em 
diferentes áreas, sendo que alguns podem estar despreparados.

A vida universitária impõe aos estudantes uma série de mudanças e exigências que podem ter impacto na sua qualidade de vida. Para o universitário típico, ou seja, aquele que concluiu o ensino médio recentemente e que ingressa no ensino superior, a universidade constitui-se em um novo contexto que demanda adaptação, tanto nos aspectos sociais (fazer novos laços de amizade, por exemplo) quanto identitários (questionar-se sobre a congruência entre os interesses e valores pessoais e a escolha profissional realizada). Além disso, a universidade apresenta, em geral, um nível de exigência acadêmica superior ao do ensino médio, fazendo com que o aluno muitas vezes necessite desenvolver hábitos de estudo mais sistemáticos e também uma postura mais autônoma em relação ao processo de aprendizagem, o que pode ser fonte de estresse para o estudante. Compreender melhor como as vivências acadêmicas impactam o bem-estar é importante, pois pode permitir pensar intervenções que visem promover um melhor ajustamento dos estudantes ao momento de vida que corresponde à experiência universitária. Assim, esta pesquisa tem por objetivo investigar as relações que podem existir entre variáveis de vivências acadêmicas e o bem-estar subjetivo de alunos universitários. As seções a seguir apresentam os conceitos centrais do estudo.

\section{BEM-ESTAR SUBJETIVO}

Bem-estar subjetivo (BES) representa a avaliação que a própria pessoa faz sobre sua vida a partir de seus valores e critérios pessoais. Esta avaliação pode ser feita em duas dimensões. Uma é de natureza cognitiva e refere-se ao julgamento da satisfação com a vida em geral ou referenciada a domínios específicos (como a capacidade física e mental ou os relacionamentos so-ciais). E a outra é de natureza emocional e diz respeito ao equilíbrio entre afetos positivos e negativos viven-ciados pela pessoa (Diener, 2000).

Diener, Suh e Oishi (1997) definem três componentes primários do BES: satisfação global com a vida, afeto positivo (emoções e humor agradáveis) e afeto negativo (emoções e humor desagradáveis). Cada um dos três componentes pode ser subdividido. A satisfação global com a vida pode ser decomposta em satisfação com os vários domínios da vida atual, como também satisfação com o passado e com o futu- ro. Os principais domínios de satisfação identificados são trabalho, família, lazer, grupo social em que o indivíduo está inserido e até mesmo o próprio self (senso de si mesmo). $\mathrm{O}$ afeto positivo agrega sentimentos de alegria transitórios, uma sensação de prazer ativo. Já o afeto negativo, inclui emoções desagradáveis tais como angústia, medo, raiva, culpa, entre outros; estes estados também são momentâneos (Diener, 1995).

O BES é um campo de estudos relacionado à saúde psicológica positiva que, segundo Diener, Suh e Oishi (1997), apresenta três características importantes. Em primeiro lugar, o bem-estar não foca em estados psicológicos indesejáveis e sim nos desejáveis, o que significa que as diferenças individuais relacionadas ao sentimento de bem-estar são consideradas relevantes de serem investigadas. O campo de estudo está interessado nos fatores que diferenciam as pessoas ligeiramente felizes, das moderadamente felizes e das extremamente felizes. A segunda característica preconiza que o BES é definido através das experiências internas do indivíduo, ou seja, ele é medido a partir da perspectiva do próprio indivíduo. A terceira característica é que a pesquisa sobre BES está interessada nos sentimentos de bem-estar de longo prazo, não apenas o humor momentâneo. O que pode produzir felicidade num momento pode não ser o mesmo que produz o BES de longo termo. Embora o humor dos indivíduos possa flutuar em função dos eventos de vida, o investigador do BES está interessado no humor ao longo do tempo e não apenas em emoções passageiras (Diener, Suh, \& Oishi, 1997).

Pesquisas sobre as relações entre personalidade e o BES revelam que características de personalidade estão associadas à maneira das pessoas reagirem a eventos e situações (Diener, 1984). Muitos pesquisadores têm buscado demonstrar a relação entre traços de personalidade e outras características pessoais com o BES (Tellegen, Lynkken, Bouchard, Wilcox, Segal, \& Rich, 1988; Emmons, 1986, 1992; Magnus, Diener, Fujita, \& Pavot, 1993; Moskowitz \& Cote, 1995; DeNeve \& Cooper, 1998; Emmons \& McCullough, 2003; Steel, Schmidt, \& Schultz, 2008).

A personalidade tem sido considerada um dos mais fortes e consistentes preditores do BES (Diener et al., 1992; 2000). Alguns autores sustentam que as pessoas apresentam uma predisposição genética para sentirem-se mais ou menos felizes (por exemplo, Tellegen 
et al., 1988). Já outros pesquisadores enfatizam o conjunto de características de personalidade e outras disposições cognitivas que podem estar associadas ao BES (Costa \& McCrae, 1980; Headey \& Wearing, 1989; Larsen \& Ketelaar, 1991; Hills \& Argyle, 2001).

As relações entre BES e personalidade vêm sendo estudadas principalmente dentro do modelo dos Cinco Grandes Fatores de personalidade: extroversão/introversão, socialização, realização, neuroticismo/estabilidade emocional e abertura à experiência (Costa \& McCrae, 1988). Desses cinco fatores, os que vêm apresentando correlações mais fortes e consistentes com o bem-estar subjetivo são extroversão e neuroticismo: a extroversão relaciona-se com sentir emoções mais positivas e o neuroticismo relaciona-se fortemente em experienciar emoções negativas (Diener, Sandvik, Pavot, \& Fujita, 1992; Maya, 2005, Zanon \& Hutz, 2013).

Costa e McCrae (1980) acreditam que o fator extroversão relaciona-se aos afetos positivos porque se trata de um fator constituído por facetas como sociabilidade, vigor e envolvimento social, que são características psicológicas tidas como positivas. Por outro lado, o neuroticismo é composto por características como ansiedade, hostilidade e impulsividade, o que explicaria sua relação com os afetos negativos. Conclusões semelhantes sobre a relação entre extroversão e afetos positivos e neuroticismo e afetos negativos foram também apresentados por outros pesquisadores (Diener \& Emmons, 1985; Watson, 1988; Larsen \& Ketelaar 1991).

Mais tarde, McCrae e Costa (1991) sugeriram também que socialização e realização poderiam aumentar a probabilidade de experiências positivas em situações sociais e de desempenho, o que levaria a um aumento no BES. Por outro lado, a abertura à experiência pode levar a pessoa a experimentar ambos os estados emocionais, positivos e negativos (Costa \& McCrae, 1980; McCrae \& Costa, 1991).

Hills e Argile (2001) reafirmaram a correlação entre extroversão, neuroticismo e bem-estar subjetivo; entretanto, esses autores sugeriram que extroversão e neuroticismo mais refletem o bem-estar do que o determinam. Sobre este tema, Steel et al. (2008) relatam que os resultados encontrados em seus estudos não só indicam que a personalidade está substancialmente relacionada ao BES, mas também que esta relação é tipicamente mais forte que os resultados apresentados pelas pesquisas anteriores.

\section{Bem-estar subjetivo em universitários e vivências acadêmicas}

As pesquisas sobre BES em estudantes universitários têm procurado identificar possíveis variáveis correlatas relevantes para essa população. Como se observa em outros perfis populacionais, o BES também apresenta uma forte correlação com personalidade, espe-cialmente neuroticismo (correlação negativa), extroversão e socialização (Nunes, Hutz, \& Giacomoni, 2009). Outros estudos internacionais que avaliaram amostras universitárias têm revelado que a satisfação de vida em geral e a satisfação de vida acadêmica estão positivamente relacionadas com decisão de carreira, personalidade, otimismo, senso de identidade e orientação para o trabalho (Lounsbury et al., 1999; Lounsbury et al., 2005), além de rendimento acadêmico e satisfação com as atividades acadêmicas (Chow, 2005).

Segundo Bardagi (2007), existem poucas pesquisas nacionais investigando satisfação de vida em universitários, ou relacionando os índices de satisfação de vida a aspectos da experiência universitária. Na maioria dos casos, as pesquisas avaliam qualidade/satisfação de vida em alunos de uma área ou curso específico (Almeida, Pinto, \& Almeida, 2006; Oliveira, 1999; Saupe, Nietche, Cestari, Georgi, \& Krahl, 2004; entre outros). Oliveira (1999) observou que trabalhar em área relacionada ao curso universitário, levar pouco tempo para chegar à universidade e ter mais idade foram aspectos associados à melhor qualidade de vida entre alunos de Educação Física. São poucos, também, os estudos identificando fontes de satisfação vocacional/acadêmica entre universitários. Pachane (2004) apontou que os aspectos relacionados à satisfação costumam ser os relacionamentos pessoais, a aprendizagem, o crescimento pessoal, a formação profissional, a qualidade do curso e a qualidade da universidade.

Dada a centralidade que a vida universitária tem na vida de muitos estudantes (pelo menos para aqueles mais jovens que não trabalham e ocupam-se a maior parte do seu tempo com o estudo), torna-se relevante investigar se há relações entre o bem-estar subjetivo destes estudantes e diferentes aspectos de suas vivências no ensino superior. A experiência universitária vem sendo estudada principalmente através de modelos dimensionais, sendo que um dos modelos empre- 
gados em pesquisas é o do Questionário de Vivências Acadêmicas - versão reduzida (Almeida, Soares, \& Ferreira, 2002; Granado, 2004). Este instrumento, adaptado para o Brasil por Granado (2004), avalia cinco grandes dimensões de vivências no âmbito universitário: pessoal (ajustamento psicológico), interpessoal (nível de integração com amigos e percepção de apoio), carreira (decisão e satisfação com a escolha profissional), estudo (nível de organização e compromisso com estudo) e institucional (satisfação e vínculo com a instituição).

Este estudo parte da hipótese geral de que vivências acadêmicas positivas (representadas por escores elevados nas dimensões mencionadas) contribuem para o bem-estar subjetivo de estudantes universitários. Assim, espera-se que existam correlações positivas entre as vivências acadêmicas e os componentes do BES (satisfação de vida, afetos positivos e afetos negativos). Uma vez que a literatura indica efeitos importantes da personalidade sobre a BES, pretendese também investigar as relações entre as vivências acadêmicas e a personalidade (no modelo dos cinco Grandes Fatores: Neuroticismo, Extroversão, Abertura, Socialização e Realização), e por fim avaliar o quanto as cinco variáveis de vivências acadêmicas predizem a variância de afetos positivos, afetos negativos e satisfação de vida controlando o efeito dos fatores de personalidade.

\section{MÉTODO}

\section{Participantes}

Compuseram a amostra 227 universitários (59\% mulheres e $41 \%$ homens) de uma instituição pública do sul do país. Os estudantes apresentaram média de idade de 23,3 anos (DP =6,3) e participaram voluntariamente do estudo. Tomaram parte no estudo universitários dos seguintes cursos: administração $(47,6 \%)$, psicologia $(15,9 \%)$, relações publicas $(9,3 \%)$, odontologia $(6,2 \%)$, nutrição $(5,7 \%)$, enfermagem $(5,3 \%)$ e outros $(10 \%)$.

\section{Instrumentos e procedimentos}

Questionário de dados sócio-demográficos e informações acadêmicas: trata-se de um questionário desenvolvido para caracterizar a amostra.

Questionário de Vivências Acadêmicas - Revisado (QVA-r; Granado, 2004): é um instrumento de autorrelato reduzido baseado em uma versão mais extensa originalmente desenvolvida em Portugal por Almeida, Soares e Ferreira (2002). Avalia cinco grandes dimensões de vivências no âmbito universitário: pessoal, interpessoal, carreira, estudo e institucional. A adaptação brasileira do QVA-r foi desenvolvida por Granado (2004), tendo apresentado boa validade fatorial e bons índices de consistência interna.

Marcadores Reduzidos da Personalidade (HauckFilho, Machado, Teixeira, \& Bandeira, 2012): trata-se de um conjunto de 25 adjetivos descritores da personalidade dentro do modelo dos Cinco Grandes Fatores: Socialização, Extroversão, Realização, Neuroticismo e Abertura à Experiência. É uma versão reduzida de um instrumento maior desenvolvido por Hutz, Silveira, Serra, Anton e Wieczorek (1998). A versão reduzida apresentou evidências de validade por análise fatorial e índices aceitáveis de consistência interna (Hauck-Filho et al., 2012).

Escala de Satisfação de Vida (Diener et al., 1985, adaptada inicialmente por Hutz e Giacomoni, 1997, e mais recentemente por Zanon, Bardagi, Layous e Hutz, 2013 para universitários): é uma escala de autorelato de cinco itens (com sete pontos), e fornece uma medida da avaliação cognitiva global da satisfação com a vida. A escala apresenta consistência interna adequada $(\alpha=0,91)$.

Escala de Afetos Positivos e Negativos (Watson \& Clarck, 1994; adaptado inicialmente por Hutz e Giacomoni, 1997, e mais recentemente por Hutz e Zanon, 2010 para universitários): consiste de 20 itens (adjetivos), metade deles relacionados a afetos positivos e a outra metade a afetos negativos. Os sujeitos respondem em uma escala de 5 pontos o quanto têm vivenciado cada um dos traços emocionais.

\section{Procedimentos}

As coordenações dos cursos foram contatadas, por conveniência do pesquisador, e a proposta da pesquisa foi apresentada. Nos cursos em que houve interesse em colaborar na pesquisa, o agendamento da aplicação dos questionários em sala de aula foi feito conforme disponibilidade. A aplicação dos questionários consistiu numa explicação dos objetivos da pesquisa aos estudantes e convite para participação na mesma (ressaltando-se o caráter anônimo e voluntário da pesquisa), seguida da leitura e obtenção de um Termo de Consentimento Livre e Esclarecido junto aos alunos, antes da aplicação dos questionários. 


\section{Análise dos dados}

Foram calculados escores para cada uma das variáveis de interesse da pesquisa. Após isso, todas as variáveis foram correlacionadas entre si, verificandose as correlações bivariadas. Posteriormente, foram realizadas análises de regressão múltipla, tendo como variáveis-critério os componentes do BES (satisfação de vida, afetos positivos e afetos negativos) e como preditoras as variáveis de personalidade e de vivências acadêmicas.

\section{RESULTADOS}

Inicialmente foram avaliadas as médias, desviospadrão e coeficientes alfa das medidas utilizadas no estudo (Tabela 1). Como pode ser observado, a maioria das medidas apresentou consistência interna satisfatória, exceto o fator Abertura (personalidade) e a dimensão Institucional (vivências acadêmicas). Estes resultados indicam que os instrumentos avaliaram adequadamente os participantes em relação ao nível de personalidade, bem-estar subjetivo e vivências acadêmicas. Os baixos coeficientes alfa verificados no fator Abertura e na dimensão Institucional sugerem que estes construtos foram avaliados com consideráveis níveis de erro de medida e devem ser interpretados com cautela.

\begin{tabular}{|c|c|c|c|c|}
\hline Variáveis & $\begin{array}{c}\text { Amplitude } \\
\text { máx. possível }\end{array}$ & M & DP & $\begin{array}{c}\text { Coeficiente } \\
\text { Alfa }\end{array}$ \\
\hline \multicolumn{5}{|l|}{ Personalidade } \\
\hline Extroversão & $5-25$ & 16,8 & 4,3 & 0,85 \\
\hline Realização & $5-25$ & 19,9 & 3,2 & 0,78 \\
\hline Socialização & $5-25$ & 20,0 & 2,9 & 0,74 \\
\hline Neuroticismo & $5-25$ & 12,2 & 4,0 & 0,77 \\
\hline Abertura & $5-25$ & 15,6 & 3,7 & 0,60 \\
\hline \multicolumn{5}{|l|}{ Bem-estar subjetivo } \\
\hline Satisfação de vida & $5-35$ & 24,8 & 5,6 & 0,82 \\
\hline Afeto positivo & $10-50$ & 35,5 & 5,9 & 0,79 \\
\hline Afeto negativo & $10-50$ & 22,5 & 6,7 & 0,84 \\
\hline \multicolumn{5}{|l|}{ Vivências acadêmicas } \\
\hline Pessoal & $13-65$ & 46,1 & 9,5 & 0,85 \\
\hline Interpessoal & $13-65$ & 44,6 & 8,1 & 0,87 \\
\hline Carreira & $13-65$ & 45,9 & 6,7 & 0,79 \\
\hline Estudo & $13-65$ & 30,1 & 6,0 & 0,77 \\
\hline Institucional & $8-40$ & 31,7 & 4,2 & 0,66 \\
\hline
\end{tabular}

Posteriormente, foram realizadas correlações de Pearson entre as variáveis estudadas (Tabela 2). Uma vez que o objetivo central do trabalho foi verificar as relações do bem-estar subjetivo com a personalidade e as vivências acadêmicas, os comentários a seguir realçam as correlações verificadas entre essas variáveis. Satisfação de vida apresentou correlações mais expressivas com a dimensão Pessoal e Neuroticismo, o que indica que participantes com níveis mais elevados de satisfação de vida também apresentam níveis elevados de vivências acadêmicas de âmbito pessoal e apresentam baixos escores de Neuroticismo. Afeto Positivo exibiu associações mais fortes com a dimensão Pessoal e Extroversão (positivas) e Neuroticismo (negativa). Estes resultados mostram que estudantes com altos níveis de afeto positivo também vivenciam 
mais experiências satisfatórias de caráter pessoal e baixos níveis de traços que compõem o fator Neuroticismo. Por fim, Afeto Negativo mostrou-se também mais fortemente correlacionado com a dimensão Pessoal e Extroversão (negativamente) e Neuroticismo (positivamente). Isto indica que quanto mais emoções negativas são vivenciadas, maior grau de neuroticismo apresentam os estudantes e menor é a qualidade das vivências acadêmicas pessoais, assim como os níveis de extroversão.

Algumas correlações entre as vivências acadêmicas e personalidade também merecem destaque. A dimensão Pessoal correlacionou-se forte e negativamente com Neuroticismo, sugerindo que se trata de construtos muito similares (embora com sentidos inversos). A dimensão Interpessoal, como se poderia esperar, teve correlações moderadas com Extroversão e Socialização, indicando que quanto maior o nível de vivências interpessoais satisfatórias, maior a expressão de traços de personalidade relacionados a Extroversão e Socialização. Por sua vez, as dimensões Carreira e Estudo mostraram correlações mais fortes com o fator Realização (mais fraca no caso da Carreira e mais forte no caso de Estudo). Estes resultados informam que quanto maior a manifestação de traços associados ao fator Realização, maior o nível de experiências acadêmicas relacionadas à dimensão Estudo e Carreira. Por fim, a dimensão Institucional apresentou um padrão global de relacionamento mais fraco com a personalidade (as correlações foram apenas fracas para Extroversão, Socialização e Neuroticismo), o que indica que as vivências acadêmicas relacionadas à Instituição estão muito pouco relacionadas com os fatores Extroversão, Socialização e Neuroticismo.

Tabela 2

Correlações entre as variáveis do estudo

\begin{tabular}{|c|c|c|c|c|c|c|c|c|c|c|c|c|c|}
\hline & (1) & (2) & (3) & (4) & (5) & (6) & (7) & $(8)$ & (9) & (10) & (11) & (12) & (13) \\
\hline 1. Pessoal & - & & & & & & & & & & & & \\
\hline 2. Interpessoal & 31 & - & & & & & & & & & & & \\
\hline 3. Carreira & 44 & 26 & - & & & & & & & & & & \\
\hline 4. Estudo & 37 & 16 & 40 & - & & & & & & & & & \\
\hline 5. Instituicional & 23 & 20 & 45 & 34 & - & & & & & & & & \\
\hline 6. Satisfação de vida & 49 & 36 & 32 & 32 & 29 & - & & & & & & & \\
\hline 7. Extroversão & 35 & 40 & 27 & 18 & 07 & 28 & - & & & & & & \\
\hline 8. Socialização & 23 & 39 & 22 & 20 & 29 & 31 & 14 & - & & & & & \\
\hline 9. Realização & 32 & 19 & 34 & 67 & 28 & 29 & 13 & 36 & - & & & & \\
\hline 10. Abertura & 12 & -00 & 09 & -01 & 03 & 03 & 22 & 08 & -07 & - & & & \\
\hline 11. Neuroticismo & -77 & -31 & -33 & -30 & -24 & -46 & -39 & -14 & -25 & 00 & - & & \\
\hline 12. Afeto positivo & 49 & 34 & 37 & 26 & 25 & 41 & 41 & 38 & 31 & 39 & -45 & - & \\
\hline 13. Afeto Negativo & -73 & -30 & -27 & -27 & -22 & -48 & -46 & -19 & -22 & -07 & 79 & -42 & - \\
\hline
\end{tabular}

Nota: o valor inteiro das correlações (zero-vírgula) foi omitido para economia de espaço. Correlações maiores ou iguais a 0,18 são significativas ao nível de 0,01; entre 0,14 e 0,16 ao nível de 0,05; menores do que 0,14 não são estatisticamente significativas.

Três análises de regressão múltipla foram realizadas tendo como variáveis-critério satisfação de vida, afetos positivos e afetos negativos e como variáveis preditoras os cinco fatores da personalidade e as cinco dimensões de satisfação acadêmica. Ou seja, estas análises foram realizadas para avaliar o poder preditivo dos fatores de personalidade e vivências acadêmicas sobre o bem-estar subjetivo. Utilizou-se um método hierárquico em que no primeiro passo foram intro- duzidos os fatores da personalidade e no segundo passo foram introduzidas as cinco dimensões de satisfação acadêmica. Este método possibilita conhecer o percentual de variância exclusiva que as vivências acadêmicas contribuem para o bem-estar subjetivo para além da contribuição dos fatores de personalidade. Em outras palavras, estas análises tiveram por objetivo avaliar o quanto de variância seria predito pelas variáveis de satisfação acadêmica, controlando o 
que já foi explicado pelos fatores de personalidade. A Tabela 3 apresenta os três modelos de regressões.

Em relação ao primeiro modelo, pode-se observar que os cinco grandes fatores da personalidade predisseram aproximadamente $27 \%$ da variância da satisfação de vida (passo 1). Com a inclusão das variáveis referentes à adaptação acadêmica (passo 2), esse percentual passou para 33\%, o que indica que essas variáveis acrescentam $6 \%$ na predição da variância total de satisfação de vida. Também se verificou que, no passo 2, somente as variáveis Extroversão, Pessoal e Interpessoal apresentaram coeficientes Beta significativos. Ou seja, Extroversão é a variável mais relevante dos fatores de personalidade no modelo, enquanto apenas as dimensões Pessoal e Interpessoal foram relevantes para predição de satisfação de vida. Ademais, os fatores de personalidade foram responsáveis pela maior parcela de predição de satisfação de vida.

Tabela 3

Modelos de regressão múltipla (hierárquica) tendo com variáveis-critério satisfação de vida, afeto positivo e afeto negativo

\begin{tabular}{|c|c|c|c|c|}
\hline \multirow{3}{*}{$\begin{array}{l}\text { Variáveis preditoras no } \\
\text { modelo }\end{array}$} & & \multicolumn{3}{|c|}{ Variáveis critério } \\
\hline & & Sat. Vida & Afeto Pos. & Afeto Neg. \\
\hline & & Beta & Beta & Beta \\
\hline \multicolumn{5}{|l|}{ Passo 1} \\
\hline Extroversão & & 0,10 & $0,18^{*}$ & $-0,17^{*}$ \\
\hline Socialização & & $0,20^{*}$ & $0,23^{*}$ & $-0,09^{*}$ \\
\hline Realização & & 0,11 & $0,12^{*}$ & $-0,01$ \\
\hline Abertura & & 0,00 & $0,35^{*}$ & $-0,04$ \\
\hline \multirow[t]{2}{*}{ Neuroticismo } & & $-0,34^{*}$ & $-0,32^{*}$ & $0,70^{*}$ \\
\hline & $\mathrm{R}^{2}$ & 0,27 & 0,49 & 0,64 \\
\hline \multicolumn{5}{|l|}{ Passo 2} \\
\hline Extroversão & & $0,05^{*}$ & $0,15^{\star}$ & $-0,21^{*}$ \\
\hline Socialização & & 0,12 & $0,18^{*}$ & $-0,07$ \\
\hline Realização & & $-0,01$ & 0,10 & 0,02 \\
\hline Abertura & & $-0,01$ & $0,33^{*}$ & $-0,01$ \\
\hline Neuroticismo & & $-0,13$ & $-0,19^{*}$ & $0,48^{*}$ \\
\hline Pessoal & & $0,22^{*}$ & 0,15 & $-0,34^{*}$ \\
\hline Interpessoal & & $0,14^{*}$ & 0,08 & 0,04 \\
\hline Carreira & & 0,01 & 0,08 & $0,14^{*}$ \\
\hline Estudo & & 0,10 & $-0,05$ & 0,01 \\
\hline \multirow[t]{2}{*}{ Institucional } & & 0,09 & 0,04 & $-0,1$ \\
\hline & $\mathrm{R}^{2}$ & 0,33 & 0,52 & 0,68 \\
\hline
\end{tabular}

*betas significativos $(p<0,01)$; R2 representa a proporção de variância explicada nas variáveiscritério pelas variáveis preditivas.

Em relação ao segundo modelo, pode-se observar que os cinco grandes fatores da personalidade predisseram aproximadamente $49 \%$ da variância de afetos positivos (passo 1). Com a inclusão das variáveis referentes à adaptação acadêmica (passo 2), esse percentual passou para $52 \%$, o que indica que essas variáveis acrescentam apenas 3\% na capacidade de predição da variância total de afetos positivos. Também se verifi- cou que, no passo 2, apenas as variáveis Extroversão, Socialização, Abertura e Neuroticismo apresentaram coeficientes Beta significativos. Estes resultados indicam que as variáveis relacionadas à adaptação acadêmica são insignificantes comparadas aos fatores de personalidade que predizem quase metade da variância de afetos positivos. 
Em relação ao terceiro modelo, pode-se observar que os cinco grandes fatores da personalidade predisseram aproximadamente $64 \%$ da variância de afetos negativos (passo 1). Com a inclusão das variáveis referentes à adaptação acadêmica (passo 2), esse percentual passou para $68 \%$, o que indica que essas variáveis acrescentam apenas $4 \%$ na capacidade de predição da variância total de afetos negativos. Também se verificou que, no passo 2, apenas as variáveis Neuroticismo, Extroversão, Pessoal e Carreira apresentaram coeficientes Beta significativos. Estes achados revelam que novamente os fatores de personalidade são os principais preditores de afetos negativos e a contribuição de variáveis associadas à adaptação acadêmica é mínima.

\section{DISCUSSÃO}

Este trabalho teve por objetivo central verificar o quanto aspectos da vivência acadêmica de universitários e a personalidade eram capazes de predizer o BES dos estudantes. Porém, antes de discutir estes resultados específicos, vale salientar as características da amostra estudada em relação aos indicadores de bemestar (satisfação de vida, afetos positivos e afetos negativos). Quando se comparam os escores obtidos neste estudo com os resultados de outras pesquisas, verifica-se uma convergência entre os estudos, indicando que estudantes universitários tendem a apresentar níveis elevados de bem-estar subjetivo, ou pelo menos de satisfação de vida (Bardagi \& Hutz, 2010).

No que diz respeito à contribuição da personalidade e das vivências acadêmicas para a predição do BES, os resultados indicaram, de acordo com a literatura e conforme esperado, que a personalidade é um importante preditor de bem-estar subjetivo (DeNeve \& Cooper, 1998; Lucas \& Diener, 2010), sendo que Neuroticismo, Socialização e Extroversão parecem ser os fatores mais relevantes para o BES (McCrae \& Costa, 1991; Steel et al., 2008). Verificou-se, ainda, que a personalidade explica muito mais a dimensão emocional do BES (afetos positivos e afetos negativos) do que a dimensão cognitiva (satisfação de vida). Tal achado sugere que a personalidade parece mais determinante para a percepção dos universitários de emoções como aflição, rancor, amabilidade ou entusiasmo do que para suas avaliações e julgamentos do quão satisfeitos eles estão com suas vidas de modo geral. Contrário às expectativas, as variáveis relacionadas às vivências acadêmicas (ou adaptação acadê- mica) não contribuíram muito para a predição de bemestar (entre 3 e $6 \%$ ) para além do que os fatores de personalidade já explicavam.

Embora fosse esperada uma contribuição exclusiva mais expressiva das vivências acadêmicas para os estudantes universitários, os resultados obtidos mostram que uma parcela do bem-estar dos estudantes, ainda que pequena, é provavelmente decorrente da qualidade de suas experiências na universidade. Nesse sentido, é importante notar que o efeito observado é pequeno quando se considera os efeitos exclusivos das vivências acadêmicas, controlando-se os efeitos da personalidade. Ao verificarmos individualmente as correlações bivariadas entre as vivências acadêmicas e os componentes do BES, percebem-se valores um pouco mais elevados, entre 0,22 e 0,73 , indicando que vivências acadêmicas encontram-se consideravelmente associadas com satisfação de vida e afetos. As correlações mais altas $(0,49$ e $-0,77)$ são com a dimensão Pessoal, cujo significado aproxima-se muito da própria definição de Neuroticismo. Questiona-se, assim, até que ponto a dimensão Pessoal do QVA-r constituise numa medida de ajustamento pessoal ao contexto acadêmico ou se é uma medida generalizada de ajustamento emocional (o que parece ser mais o caso). Uma análise do conteúdo dos itens do instrumento (que não se referem especificamente ao contexto acadêmico) e a alta correlação observada com Neuroticismo $(-0,77)$ levam a crer que a dimensão Pessoal do QVA-r nada mais é do que uma medida de ajustamento emocional geral. Estudos futuros talvez possam investigar melhor a pertinência de se utilizar a dimensão Pessoal do QVA-r como variável relevante para pesquisas sobre adaptação de universitários, na medida em que esta dimensão se confunde com o traço de Neuroticismo.

As demais dimensões de vivências acadêmicas, no entanto, não parecem se confundir com traços de personalidade, e ainda assim apresentaram correlações com BES entre 0,22 e 0,37. Estas correlações indicam uma tendência de que vivências acadêmicas mais satisfatórias possam produzir mais bem-estar nos estudantes. A dimensão Carreira, que envolve estar identificado e satisfeito com a escolha profissional, apresentou correlação de 0,32 com Satisfação de vida e 0,37 com Afeto Positivo. Este resultado é semelhante ao observado por Lounsbury al. (1999), que verificaram uma correlação de 0,43 entre decisão de carreira (um construto relacionado à identificação com a carreira) e 
satisfação de vida. Estes achados indicam que a confiança e satisfação com a escolha profissional pode trazer mais emoções positivas para a vida dos estudantes e também avaliações mais positivas de suas vidas. Do mesmo modo, Bardagi e Hutz (2010), utilizando a mesma escala de satisfação de vida empregada neste estudo, observaram correlações da ordem de 0,20 entre satisfação de vida e satisfação com a escolha da profissão. Estes dados sugerem que, quanto mais seguros da escolha profissional os acadêmicos se sentem, mais elevada é a percepção de satisfação de vida.

Com relação à dimensão Interpessoal, que envolve o relacionamento com os colegas, incluindo estabelecimentos de amizades e percepção de habilidades sociais, verificou-se uma correlação de 0,36 com Satisfação de vida e 0,34 com Afeto Positivo. Tais correlações indicam que vivências interpessoais mais satisfatórias dos estudantes podem produzir mais prazer, entusiasmo e contentamento com a vida de forma geral. De acordo com Diener e Seligman (2002), ter estreitas relações pessoais com os outros contribui significativamente para a felicidade. Isto sugere que a capacidade de construir relacionamentos pessoais próximos pode ter um profundo impacto no bem-estar. Percebe-se, assim, a importância das relações interpessoais no contexto da universidade para os estudantes. Para o aluno típico (jovem que ingressa no ensino superior logo após o ensino médio ou pouco tempo depois), a universidade constitui-se num contexto de desenvolvimento psicossocial de relevância. É lá que novas amizades são construídas, permitindo a troca de experiências com os pares e o desenvolvimento de uma rede de suporte emocional fora da família, o que deve contribuir para a percepção de bem-estar subjetivo.

A dimensão Estudo, que envolve competências, hábitos de estudo e organização do estudo, apresentou correlação de 0,32 com Satisfação de Vida e 0,25 com Afeto Positivo, o que sugere que a adequação às demandas da universidade pode contribuir positivamente para o surgimento de emoções como segurança, vigor e determinação. Alunos que conseguem se organizar frente às exigências acadêmicas e respondê-las a contento provavelmente apresentam melhor desempenho e, possivelmente, derivam sua satisfação da percepção de que estão obtendo sucesso no caminho escolhido. De fato, há evidências de que existe uma relação positiva entre o desempenho acadêmico e a satisfação com a vida (Powers, 2008). Dada a centralidade que o papel de estudante possui na vida de grande parte dos universitários, faz sentido que uma percepção de estar conseguindo se desempenhar bem nesse papel esteja positivamente associado ao bem-estar.

Já a dimensão Institucional, que significa satisfação e vínculo com a instituição, teve uma correlação de 0,29 com Satisfação de Vida e 0,25 com Afeto Positivo, indicando pouca associação entre satisfação com a instituição e satisfação de vida e afetos positivos. Esta dimensão apresentou as correlações mais baixas com os indicadores de bem-estar, sugerindo ser a que menos impacto tem sobre estas variáveis. Devido à baixa consistência interna da dimensão Institucional $(0,60)$, o que evidencia mais erros de medida, é possível que essas correlações estejam submestimadas. Contudo, ainda assim foram estatisticamente significativas. Por isso, é razoável supor que a satisfação com o ambiente no qual o estudante passa boa parte do seu tempo, bem como aquilo que significa para o estudante estar estudando na instituição com a qual tem vínculo, sejam aspectos que contribuem para uma percepção de satisfação mais geral com a vida. Isso pode ser especialmente o caso quando a universidade é a instituição de escolha do aluno, o que deve ter sido o caso nesta pesquisa, já que se tratava de uma universidade pública bem conceituada.

Porém, uma vez que a personalidade parece ser o grande responsável pela predição de BES, e que as dimensões da satisfação acadêmica acrescentam pouca variância exclusiva, parece plausível pensar que baixos níveis de bem-estar em universitários devem-se mais a uma predisposição à ansiedade, depressão, entre outras características psicológicas de cunho negativo, do que propriamente a dificuldades na adaptação acadêmica. De fato, é possível que a menor qualidade nas relações interpessoais, insatisfação com a escolha de curso, dificuldades de adaptação, insatisfação com a instituição e pessimismo em relação às perspectivas futuras de carreira sejam influenciadas por altos níveis de Neuroticismo, baixa Socialização e Extroversão. Já que a personalidade pode de alguma forma influenciar a forma como as pessoas colorem e interpretam eventos de vida (McCrae \& Costa, 2010), parece razoável supor que estudantes emocionalmente instáveis, mais tímidos e com menores habilidades sociais avaliem o ambiente como sendo mais hostil e com menos oportunidades, ao mesmo tempo em que não estabelecem redes de apoio que possam ajudar a modificar essa situação. Pessoas em tal situação provavelmente tendem a julgar o seu comportamento e 
realizações como estando aquém de suas expectativas, o que os leva a uma avaliação de baixo bem-estar.

Resultados semelhantes foram verificados por Lounsbury et al. (2005). Os autores sugerem que quem os estudantes se tornam quando entram no meio acadêmico, assim como quão satisfeitos eles se sentem com diferentes aspectos da experiência universitária, poderia ser explicado por quem eles eram quando entraram na universidade. Em outras palavras, a personalidade (que é estável e associada à satisfação) pode mudar muito pouco no período de tempo que antecede a entrada na universidade até o meio ou fim do curso. Logo, ela pode ser a responsável pelos níveis de bem-estar subjetivo dos universitários, enquanto a qualidade da experiência acadêmica pode ser uma consequência da personalidade dos estudantes.

As implicações de tais achados, ainda que preliminares, apontam para a importância de se avaliar a personalidade quando se pensa em intervenções voltadas a estudantes universitários que visem promover $\mathrm{o}$ bem-estar e reduzir problemas de adaptação ao contexto acadêmico. Há sugestões, na literatura, de que os clínicos de saúde mental rotineiramente administrem uma escala de cinco fatores de personalidade para determinar se avaliações mais extensas de transtornos de personalidade relacionadas com neuroticismo e outros traços necessita ser conduzida (Widiger \& Trull, 2007). Por exemplo, estudantes com características muito marcadas de Neuroticismo talvez necessitem de intervenções mais individualizadas e com um foco mais clínico. Já outros alunos, sem traços de desajustamento psicológico mais geral, podem se beneficiar de intervenções mais pontuais, voltadas ao desenvolvimento de habilidades sociais ou de hábitos de estudo.

\section{CONSIDERAÇÕES FINAIS}

Por fim, deve-se apontar que o delineamento correlacional deste estudo não permite inferir efeitos causais entre as variáveis. Por exemplo, é plausível supor que tanto o Neuroticismo possa levar a pessoa a apresentar uma baixa adaptação no Estudo, quanto que uma baixa adaptação na dimensão Estudo também pode produzir as reações que caracterizam o Neuroticismo.

Verificou-se também que a variável Neuroticismo e a dimensão Pessoal do QVA-r se sobrepõem conceitualmente. Isso se torna mais evidente quando se ana- lisam os conteúdos das questões da dimensão Pessoal do QVA-r. Os itens do QVA-r referem-se a ajustamento psicológico, enquanto o Neuroticismo avalia o desajustamento psicológico. Talvez a dimensão pessoal do QVA-r pudesse ser reformulada especificamente para questões acadêmicas, pois a mesma encontra-se muito vaga no instrumento (por exemplo: "Sinto-me triste ou abatido (a)").

De uma forma geral, portanto, este estudo mostrou que tanto a personalidade quanto as vivências acadêmicas apresentam relação com o bem-estar subjetivo dos estudantes universitários, embora a associação com a personalidade seja muito mais evidente. Isso não significa, contudo, que as vivências acadêmicas não sejam relevantes para o bem-estar, como mostraram as correlações. No entanto, novas pesquisas são necessárias a fim de tornar mais claras as relações entre as vivências acadêmicas e a personalidade, o que pode servir para orientar intervenções voltadas ao público universitário.

\section{REFERÊNCIAS}

Albuquerque, A. S., \& Tróccoli, B. T. (2004). Desenvolvimento de uma Escala de Bem-Estar Subjetivo. Psicologia: Teoria e Pesquisa, 20(2), 153-164.

Almeida, L. S., \& Soares, A. P. (2004). Os estudantes universitários: Sucesso escolar e desenvolvimento psicossocial. Em E. Mercuri \& S. A. J. Polydoro, (Orgs.), Estudante universitário: Características e experiências de formação (pp. 15-40). Taubaté: Cabral.

Almeida, L. S., Soares A. P., \& Ferreira, J. A. (2002). Questionário de Vivências Acadêmicas (QVA-r): Avaliação do ajustamento dos estudantes universitários. Avaliação Psicológica, 2, 81-93.

Almeida, T. L., Pinto, S. S., \& Almeida, C. I. R. (2006). Medida de satisfação dos estudantes do curso de engenharia civil da FURG: o curso sob a ótica dos estudantes em 2001 e em 2005. In: XXXIV COBENGE, 2006. Anais do XXXIV COBENGE. Passo Fundo: Ed. Universidade de Passo Fundo.

Andrews, F. M., \& Withey, S. B. (1976). Social indicators of wellbeing: American perception of life quality. New York: Plenum.

Bardagi, M. P. (2007). Evasão e comportamento vocacional de universitários: estudos sobre o desenvolvimento de carreira na graduação. Tese de doutorado não publicada. Programa de Pós-Graduação em Psicologia, Universidade Federal do Rio Grande do Sul.

Bardagi, M. P., \& Hutz, C. S. (2010). Satisfação de vida, comprometimento com a carreira e exploração vocacional em estudantes universitários. Arquivos Brasileiros de Psicologia, 62(1), 159-170. 
Chow, H. P. H. (2005) Life satisfaction among university students in a Canadian prairie city: A multivariate analysis. Social Indicators Research, 70(2), 139-150.

Costa, P. T., \& McCrae, R. R. (1980). Influence of extraversion and neuroticism on subjective well-being: Happy and unhappy people. Journal of Personality and Social Psychology, 38, 668678.

Costa, P. T., \& McCrae, R. R. (1988). Personality in adulthood: A six years longitudinal study of self-reports and spouse ratings on the NEO Personality Inventory. Journal of Personality and Social Psychology, 54, 853-863.

DeNeve, K. M., \& Cooper, H. (1998). The happy personality: A meta-analysis of 137 personality traits and subjective wellbeing. Psychological Bulletin, 124, 197-229.

Diener, E. (1984). Subjective well-being. Psychological Bulletin, 95, 542-575.

Diener, E. (1995). A value based index for measuring national quality of life. Social Indicators Research, 36, 107-127.

Diener, E. (2000). Subjective well-being: The science of happiness and a proposal for a national index. American Psychologist, 55(1), 34-43.

Diener, E., \& Emmons, R. A. (1985). The independence of positive and negative affect. Journal of Personality and Social Psychology, 47, 1105-1117.

Diener, E., \& Seligman, M. E. P. (2002). Very happy people. Psychological Science, 13, 81-84.

Diener, E., Emmons, R.A., Larsen, R.J., \& Griffin, S. (1985). The satisfaction with life scale. Journal of Personality Assessment, $49,71-75$

Diener, E., Sandvik, E., Pavot, W., \& Fujita, E. (1992). Extraversion and subjective well-being in a U.S. national probability sample. Journal of Research in Personality, 26, 205-215.

Diener, E., Suh, E. M., \& Oishi, S. (1997). Recent findings on subjective well-being. University of Illinois. Retirado de http://www.psych.uiuc.edu/ ediener/hottopic/ paper12.htm em Mar 2011.

Diener, E., Suh, E. M., Lucas, R. E., \& Smith, H. L. (1999). Subjective well-being: Three decades of progress. Psychological Bulletin, 125, 276-302.

Emmons, R. A. (1986). Personal strivings: An approach to personality and subjective well-being. Journal of Personality and Social Psychology, 51, 1058-1068.

Emmons, R. A. (1992). Abstract versus concrete goals: Personal striving level, physical illness, and psychological well-being. Journal of Personality and Social Psychology, 62, 292-300.

Emmons, R. A., \& McCullough, M. E. (2003). Counting blessings versus burdens: An experimental investigation of gratitude and subjective well-being in daily life. Journal of Personality and Social Psychology, 84(2), 377-389.

Granado, J. I. F. (2004). Vivência acadêmica de universitários brasileiros: Um estudo de validade e precisão do QVA-r. Dissertação de mestrado não-publicada, Universidade São Francisco, Itatiba.
Hauck-Filho, N., Machado, W. L., Teixeira, M. A. P., \& Bandeira, D. R. (2012). Evidências de validade de marcadores reduzidos para a avaliação da personalidade no modelo dos Cinco Grandes Fatores. Psicologia: Teoria e Pesquisa, 28, 417-423.

Headey, B., \& Wearing, A. (1989). Personality, life events, and subjective wellbeing: Toward a dynamic equilibrium model. Journal of Personality and Social Psychology, 57, 731-739.

Hills, P., \& Argyle, M. (2001). Happiness, introversion-extraversion and happy introverts. Personality and Individual Differences, 30, 595-638.

Hutz, C. S., \& Giacomoni, C. H. (1997). A mensuração do BemEstar Subjetivo: Escala de Afeto Positivo e Negativo e Escala de Satisfação de Vida [Resumo]. Trabalho apresentado na XXVI Congresso Interamericano de Psicologia, São Paulo.

Hutz, C. S., \& Zanon, C. (2012). Instrumentos para avaliação em psicologia positiva. Manuscrito em preparação.

Hutz, C. S., Silveira, A. D., Serra, J. G., Anton, M. C., \& Wieczorek, L. S. (1998). O desenvolvimento de marcadores para a avaliação da personalidade no Modelo dos Cinco Grandes Fatores. Psicologia. Reflexão e Crítica, 11, 395-415.

Larsen, R. J., \& Ketelaar, T. (1991). Personality and susceptibility to positive and negative emotional states. Journal of Personality and Social Psychology, 61, 132-140.

Lemos, T. H. (2010). Escala de avaliação da vida acadêmica: Estudo de validade com universitários da Paraíba. Universidade São Francisco, Itatiba.

Lounsbury, J. W., Saudargas, R. A., Gibson, L. W., \& Leong, F. T. (2005). An investigation of broad and narrow personality traits in relation to general and domain-specific life satisfaction of college students. Research in Higher Education, 46(6), 707729.

Lounsbury, J. W., Tatum H. E., Chambers, W., Owens, K. S., \& Gibson, L. W. (1999). An investigation of career decidedness in relation to 'big five' personality constructs and life satisfaction. College Student Journal, 33(4), 646-652.

Lucas, R. E., \& Diener, E. (2010). Personality and subjective wellbeing. In O. P. John, R. W. Robins and L. A. Pervin, (Eds). Handbook of personality. (pp. 795-814). New York: The Guilford Press. Third Edition

Lucas, R., Diener, E., \& Suh, E. (1996). Discriminat validity of well-being measures. Journal of Personality and Social Psychology, 71, 616-628.

Magnus, K., Diener, E., Fujita, F., \& Pavot, W. (1993). Extraversion and neuroticism as predictors of objective life events: A longitudinal analysis. Journal of Personality and Social Psychology, 65, 1046-1053.

Maya, T. (2005). Don't worry, be happy? Neuroticism, trait consistent affect regulation, and performance. Journal of Personality and Social Psychology, 89, 449-461.

McCrae, R. R., \& Costa, P. T. J. (2010). The five-factor theory of personality. In O. P. John, R. W. Robins and L. A. Pervin, (Eds). Handbook of personality: Theory and research. (pp. 159181). New York: The Guilford Press. ( $3^{\text {rd }}$ ed.). 
McCrae, R., \& Costa, P. T. (1991). Adding liebe und arbeit: The full five-factor model and well-being. Personality and Social Psychology Bulletin, 17(2), 227-232.

Moskowitz, D. S., \& Cote, S. (1995). Do interpersonal traits predict affect? A comparison of three models. Journal of Personality and Social Psychology, 69, 915-924.

Nunes, C. H. S., Hutz, C. S., \& Giacomoni, C. H. (2009). Associação entre bem-estar subjetivo e personalidade no modelo dos Cinco Grandes Fatores. Avaliação Psicológica, 8(1), 99-108.

Oliveira, J. A. C. (1999). Qualidade de vida em estudantes universitários de educação física. Dissertação de mestrado não publicada. Universidade Estadual de Campinas. Campinas.

Pachane, G. G. (2004). A experiência universitária e sua contribuição ao desenvolvimento pessoal do aluno. In: E. Mercuri \& S. A. Polydoro (Orgs.). Estudante universitário: Características e experiências de formação (pp. 155-186). Taubaté, São Paulo: Cabral Ed. e Livraria Universitária.

Powers, C. I. (2008). Academic achievement and social involvement as predictors of life satisfaction among college students. Psi Chi Journal on Undergraduate Research, 13, 128-135.

Santos, L., \& Almeida, L. A. (2001). Vivências acadêmicas e rendimento escolar: Estudo com alunos universitários do $1^{\circ}$ ano. Análise Psicológica, 2(19), 205-217.

Saupe, R., Nietche, E. A., Cestari, M. E., Georgi, M. D. M., \& Krahl, M. (2004). Qualidade de vida dos acadêmicos de enfermagem. Revista Latino-americana de Enfermagem, 12(4), 636642 .

Steel, P., Schmidt, J., \& Shultz, J. (2008). Refining the relationship between personality and subjective well-being. Psychological Bulletin, 134(1), 138-161.
Tellegen, A., Lykken, D., Bouchard, T. J., Wilcox, K. J., Segal, N. J., \& Rich, S. (1988). Personality similarity in twins reared apart and together. Journal of Personality and Social Psychology, 54, 1031-1039.

Watson, D. (1988). Intraindividual and interindividual analyses of positive and negative affect: Their relation to health complaints, perceived stress, and daily activities. Journal of Personality and Social Psychology, 54, 1020-1030.

Watson, D., \& Clark, L. A. (1994). The PANAS-X: Manual for the positive and negative affect schedule - Expanded Form. The University of Iowa.

Widiger, T. A., \& Trull, T. J. (2007). Plate tectonics in the classification of personality disorder - Shifting to a dimensional model. American Psychologist, 62,71-83.

Zanon, C., Bardagi, M., Layous, K., \& Hutz, C. S. (2013). Validation of the Satisfaction with Life Scale to Brazilians: Evidences of measurement noninvariance across Brazil and US. Social Indicators Research. Doi: 10.1007/s11205-013-0478-5

Zanon, C., Hutz, C. S. (2013). Affective Disposition, thinking Styles, neuroticism and life satisfaction. Universitas Psychologica, 12, 403-411. 